\title{
Fístula aorto-esofágica secundária a fratura de endoprótese torácica: relato de caso
}

\author{
Aortoesophageal fistula secondary to thoracic stent-graft fracture: a case report \\ Felipe N asser ${ }^{1}$, Adnan N eser ${ }^{2}$, Jose C arlos Ingrund ${ }^{3}$, C harles Edouard Zurstrassen ${ }^{4}$, \\ Flavio de M acedo Cavaleiro R ibeiro ${ }^{4}$, Ricardo Vagner M oreira4, Elias Arcenio N eto ${ }^{4}$, \\ $M$ arcelo Calil Burihan ${ }^{5}, 0$ rlando C osta Barros $^{6}$
}

\begin{abstract}
Resumo
0 tratamento endovascular dos aneurismas aórticos torácicos tem se desenvolvido consideravelmente nos últimos anos. No entanto, complicações tardias desta nova modalidade terapêutica apenas agora estão sendo observadas e anal isadas. Fístulas aorto-esofágicas são complicações raras do tratamento endovascular dos aneurismas aórticos, sendo encontrados poucos relatos na literatura. 0 presente caso re porta um paciente com aneurisma aórtico torácico tratado há 4 anos e complicado com fratura da endoprótese. Após nova intervenção endovascular, foi observada extrusão da antiga endoprótese através do esôfago com formação de fístula. A pesar do tratamento realizado, o paciente evoluiu a óbito 9 dias após por hemorragia digestiva maciça. Aspectos técnicos e revisão de literatura são discutidos.
\end{abstract}

Palavras-chave: Aneurisma, fístula, implante de prótese.

\section{Introdução}

A terapia endoluminal foi introduzida na prática cirúrgica como uma nova modalidade terapêutica no tratamento das doenças da aorta, tais como aneurismas $^{1-5}$, dissecções ${ }^{6,7}$, úlceras aórticas penetrantes ${ }^{8,9}$, rotura traumática da aorta ${ }^{10,11}$, entreoutras. C ontudo,

1. Assistente, Departamento de Cirurgia Vascular, Hospital Santa Marcelina (HSM), São Paulo, SP. Responsável, Departamento de Radiologia Vascular e Intervencionista, HSM, São Paulo, SP.

2. Chefe, Serviço de Cirurgia Vascular, HSM, São Paulo, SP.

3. Supervisor, Serviço de Cirurgia Vascular, HSM, São Paulo, SP.

4. Cirurgiões vasculares estagiários, Departamento de Radiologia Vascular e Intervencionista, HSM, São Paulo, SP.

5. Médico assistente, Serviço de Cirurgia Vascular, HSM, São Paulo, SP.

6. Médico assistente, Serviço de Cirurgia Vascular, HSM, São Paulo, SP. Responsável, Departamento de Ecografia Vascular, HSM, São Paulo, SP.

Artigo submetido em 31.07.06, aceito em 04.12.06.

J Vasc Bras 2006:5(4):317-20.

Copyright $\odot 2006$ by Sociedade Brasileira de Angiologia e de Cirurgia Vascular.

\begin{abstract}
The endovascular treatment of thoracic aortic aneurysms has undergone considerable development over the past years. H owever, late complications of this new therapeutic modality have only recently been observed and analyzed. Aortoesophageal fistulas are rare complications of the endovascular treatment of aortic aneurysms, and there are few reports in the literature. We report a case of a patient with thoracic aortic aneurysm treated 4 years ago and with complications due to stent-graft fracture. After a new endovascular intervention, therewas extrusion of theold graft through the esophagus with fistula formation. D espite the treatment, the patient died 9 days later dueto massive digestivehemorrhage. Technical aspects and review of the literature are discussed.
\end{abstract}

Keywords: Aneurysm, fistula, graft implantation.

apesar dosaltosíndices desucesso técnico emelhora nas taxas demorbidadeemortalidade, osseusresultadosem longo prazo permanecem incertos ${ }^{12}$. Até 0 presente momento, a maioria dos eventos adversos encontra-se associada ao material protético e é manifestada principalmente através dos en dol eaks ${ }^{12}$. Complicações como lesão arterial, isquemia de membros superiores, embolização distal, acidente vascular cerebral e paraplegia também têm sido reportadas ${ }^{13}$

Fístulas aorto-esofágicas (FAE) e aorto-brônquicas (FAB) são complicações raras dos tratamentos cirúrgico e endovascular das doenças da aorta, porém quase sempre fatais na ausência de tratamento adequado, além de considerável morbidade e mortalidade ${ }^{14-18}$.

0 propósito desteartigo éo de descrever a ocorrência de extrusão para o esôfago de endoprótese previamente implantada em um paciente com aneurisma torácico da aorta descendente. 


\section{Relato do caso}

Paciente do sexo masculino, 48 anos, hipertenso e tabagista, com história de acidente vascular cerebral há 2 anos. $\mathrm{H}$ á 4 anosfoi submetido a tratamento endovascular de aneurisma aórtico torácico (AAT) em outro serviço, cuja origem do material utilizado não é de conhecimento dos autores.

0 paciente deu entrada neste serviço com história detossenão produtiva, associada a dispnéia aos médios esforços, ambasiniciadas háaproximadamente 3 meses. V inha apresentando piora progressiva do quadro, referindo, durante sua internação, dispnéia severa e ortopnéia, associadas a disfagia para alimentos sólidos.

Ao exame físico, não foi observada palidez cutânea ou cianose, sendo auscultados estertores crepitantes em bases pulmonares com ausculta cardíaca normal. N ão foram observadas alterações significativas dos sinais vitais.

A tomografia computadorizada (CT) do tórax na entrada mostrava aneurisma aórtico descendente e endoprótese fraturada em seu segmento médio-inferior contida no interior do saco aneurismático, além de compressão do brônquio fonte esquerdo. T ambém foi realizado esofagograma na internação, que mostrou compressão acentuada do esôfago causada pela endoprótese e aneurisma aórtico.

D evido ao risco iminente de rotura do aneurisma, foi realizada nova correção através de técnica endovascular, sendo implantada prótese T alent (M edtronic), culminando na exclusão do aneurisma aórtico e da endoprótese fraturada, respectivamente.

0 pacienteapresentou recuperação satisfatória após a cirurgia, com importantemelhora dos sintomase alta hospitalar no sétimo dia de pós-operatório. Dez dias após a alta, o paciente foi readmitido com queixas de fortes dores precordiais, irradiadas para a região dorsal, acompanhadas de sial orréia aguda e disfagia para líquidos. N ova tomografia revelou a manutenção da dilatação aneurismática aórtica, sem, no entanto, apresentar sinais de rotura ou vazamentos. Contudo, também foram observadas imagens de conteúdo gasoso situadas entre a nova endoprótese e o saco aneurismático. $\mathrm{N}$ a endoscopia digestiva alta, foi observada lesão ulcerada esofágica acompanhada de formação fistular para a aorta e para o brônquio fonteesquerdo. Surpreendentemente, também foi visualizada a antiga prótese fraturada, localizada no interior do saco aneurismático sem sinais de sangramento. Com o auxílio de pinças endos-

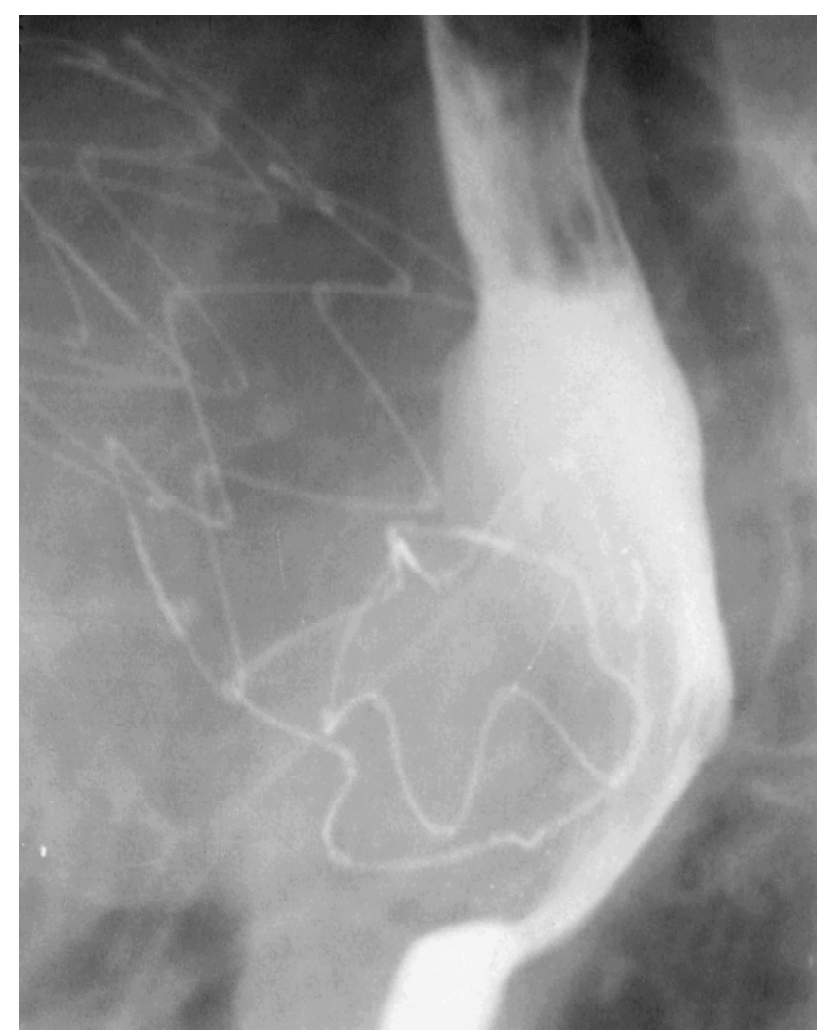

Figura 1 - Esofagograma mostrando compressão do esôfago pela prótese fraturada

cópicas, realizou-se, então, a retirada cuidadosa dos fragmentos fraturados da endoprótese. Terminada a captura, uma sonda nasoenteral foi implantada com a finalidade de aspiração e alimentação.

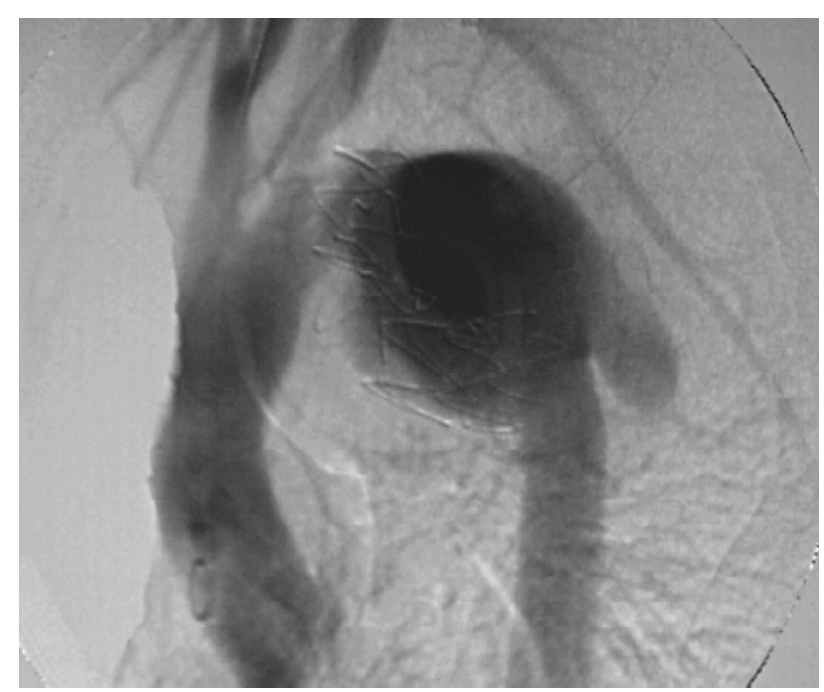

Figura 2 - Aortografiaantes do tratamento. Imagem mostrando o aneurisma aórtico recanalizado e a prótese fraturada em seu interior 
Sete dias apóso evento, foi real izada nova endoscopia, sendo encontrada cicatrização parcial da parede esofágica, estando o paciente em plena recuperação.

D ois dias após, no entanto, o mesmo apresentou episódio discreto de tosse com escarro hemoptóico, culminando com quadro dehematêmesemaciça eóbito 6 horas após.

\section{D iscussão}

Fístulas aorto-entéricas secundárias são entidades bem conhecidas e descritas, ocorrendo ocasionalmente após o reparo protético da aorta. N o entanto, de ocor-

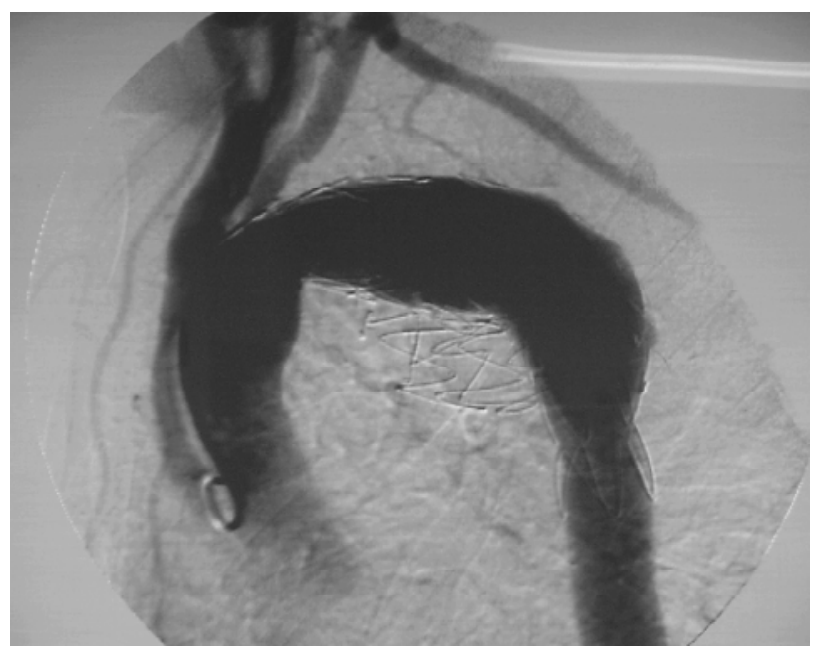

Figura 3 - Aortografia de controle após tratamento endovascular

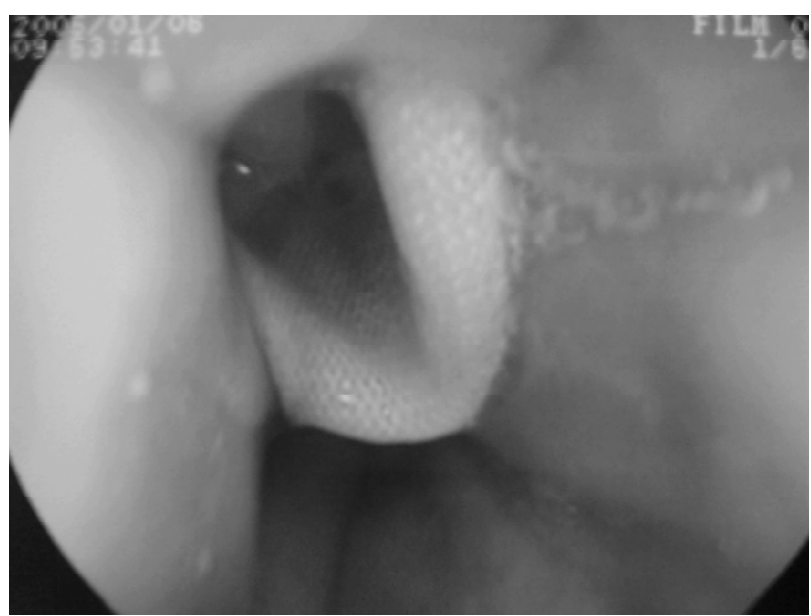

Figura 4 - Imagem endoscópica da retirada de um dos fragmentos da prótese aórtica fraturada rência mais rara, a incidência exata dasFAE secundárias ainda permanece desconhecida. Acredita-se ser bem menor do que a incidência de fístulas aorto-duodenais secundárias, estimada entre 0,4 a $4 \%$ das reconstruções abdominais aórticas ${ }^{19}$.

Em relação às FAE secundárias, poucos casos hoje estão disponíveis na literatura, sendo observada elevada mortalidade entre eles, em função não somente da presença freqüente de infecção sobre 0 material protético, mas também da alta incidência na formação de pseudo-aneurismas aórticos e sangramentos maciços. A FAE secundária também foi observada após o reparo endovascular do AAT. 0 primeiro caso foi publicado em 1998, quando Dake relatou uma série de 103 casos de aneurismas descendentes torácicos tratados com próteses endovasculares $^{13}$. N ovo caso foi publicado recentemente por $\mathrm{H}$ ance, no qual $\mathrm{O}$ autor também relata a ocorrência de FAE, 15 meses após o reparo endovascular por dissecção aguda traumática da aorta torácica ${ }^{15}$. N este caso, foi conseguida com sucesso a correção, por reparo cirúrgico aberto da aorta e do esôfago. E ggebrecht, através de análise retrospectiva de 60 pacientes tratados por reparo endovascular da aorta torácica, também observou a entidade, ocorrida em três casos $^{3}$. O s três pacientes envolvidos evoluíram para óbito, sendo o primeiro por hematêmese maciça e os outros dois, tratados de maneira conservadora, por mediastinite e sépsis.

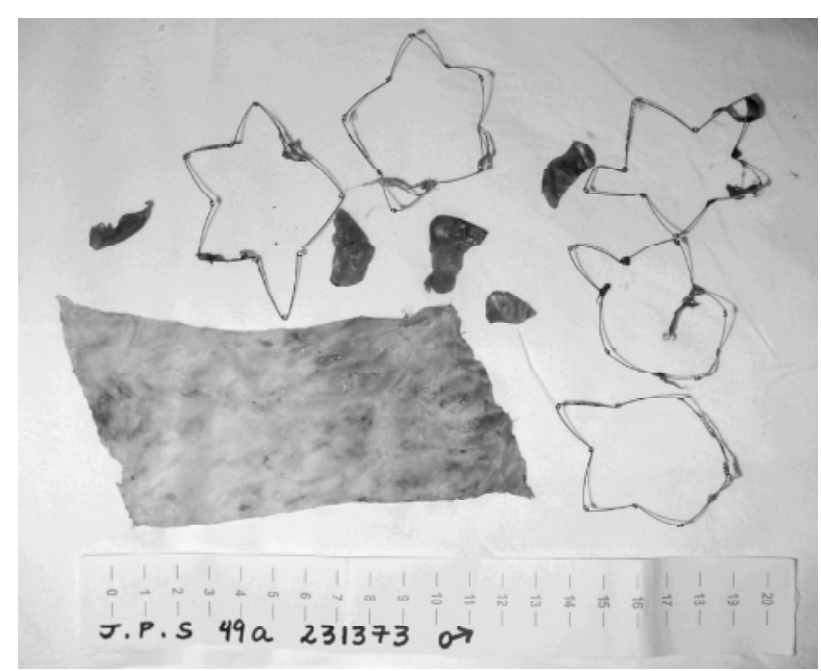

Figura 5 - Conjunto dosstentsretiradosdo trajeto fistular da aorta para o esôfago 
Dentro da sua fisiopatologia, acredita-se que as FAE observadas após terapia endoluminal ocorram devido ao desenvolvimento de pseudo-aneurismas, à presença de próteses mal seladas com vazamentos, erosão do stent através da aorta para o esôfago, ou perfuração da aorta e do esôfago através dos gan chos de fixação da endoprótese ${ }^{14}$.

0 presente caso surpreende pela ocorrência da formação fistular secundária entre a aorta e o esôfago e pela ocorrência da extrusão para o esôfago da endoprótese aórtica fraturada, sendo a mesma passível decaptura pelas pinças endoscópicas. Infelizmente, as más condições clínicas apresentadas pelo paciente impossibilitaram a realização do tratamento definitivo da FAE, constituído pelo reparo cirúrgico aberto.

Concluindo, acreditamos na necessidade de seguimentos rigorosos nos pacientes tratados por técnica endoluminal. Somente assim conseguiremos obter diagnóstico precoce dessas complicações, podendo oferecer tratamento adequado em fases mais precoces e, portanto, em melhores condições.

\section{Referências}

1. D ake M D, M iller D C, Semba CP, M itchell RS, W alker PJ, Liddell PP. Transluminal placement of endovascular stentgraftsfor thetreatment of descendingthoracic aortic aneurysms. N Engl J Med. 1994;331:1729-34.

2. Ehrlich M, Grabenwoeger M, Cartes-Zumelzu $F$, et al. Endovascular stent graft repair for aneurysmson thedescending thoracic aorta. Ann Thorac Surg. 1998;66:19-24.

3. Eggebrecht H, BaumgartD, RadeckeK, et al. Aortoesophageal fistula secondary to stent-graft repair of the thoracic aorta. J Endovasc Ther. 2004;11:161-7.

4. Temudom T, D'Ayala M, M arin ML, et al. Endovascular grafts in the treatment of thoracic aortic aneurysm and pseudoaneurysms. Ann V asc Surg. 2000;14:230-8.

5. Criado FJ, Barnatan M F, Rizk Y, Clark NS, Wang CF. Technical strategies to expand stent-graft applicability in the arch and proximal descending thoracic aorta. J Endovasc Ther. 2002;9 Suppl 2:II32-8.

6. N ienaber $C A$, Fattori $R$, Lund $G$, et al. Nonsurgical reconstruction of thoracic aortic dissection by stent-graft placement. N Engl J M ed. 1999;340:1539-45.
7. Palma JH, de Souza JA, Rodrigues Alves CM, Carvalho AC, Buffolo E. Self-expandable aortic stent-graftsfor treatment of descending aortic dissections. Ann Thorac Surg. 2002;73: 1138-41; discussion 1141-2.

8. Brittenden J, M cBrideK, M cl nnes G, GillespieI I , Bradbury $A W$. The use of endovascular stents in the treatment of penetrating ulcers of the thoracic aorta. J V asc Surg. 1999;30: 946-9.

9. Schoder $M, G$ rabenwoger $M$, H olzenbein T, etal. Endovascular stent-graft repair of complicated penetrating atherosclerotic ulcers of the descending thoracic aorta. J V asc Surg. 2002;36: 720-6.

10. O rford VP, Atkinson N R, Thomson K, et al. Blunt traumatic aortic transection: the endovascular experience. Ann Thorac Surg. 2003;75:106-11; discussion 111-2.

11. O rend KH, Pamler R, Kapfer X, Liewald F, Gorich J, SunderPlassmann L. Endovascular repair of traumatic descending aortic transection. J Endovasc Ther. 2002;9:573-8.

12. LeeJT, White RA. Current status of thoracic aortic endograft repair. Surg Clin N orth Am. 2004;84:1295-318.

13. D ake M D, M iller D C, M itchell RS, Semba CP, M oore KA, Sakai T . T he "first generation" of endovascular stent-graftsfor patients with aneurysms of the descending thoracic aorta. J ThoracC ardiovasc Surg. 1998;116:689-703; discussion 703-4.

14. $H$ ance $K A, H$ su J, Eskew $T$, H ermreck AS. Secondary aortoesophageal fistula after endoluminal exclusion because of thoracic aortic transection. J V asc Surg. 2003;37:886-8.

15. Seymour EQ. Aortoesophageal fistula as a complication of aortic prosthetic graft. AJR Am J R oentgenol. 1978;131:160-1.

16. Wong RS, Champlin A, Temes RT, Wernly JA. Aortoesophageal fistula after repair of descending aortic dissection. Ann Thorac Surg. 1996;62:588-90.

17. Luketich JD, Sommers KE, Griffith BP, et al. Successful management of secondary aortoesophageal fistula. Ann T horac Surg. 1996;62:1852-4.

18. Karmy-J ones R, LeeCA, N ichollsSC, H offer E. M anagement of aortobronchial fistula with an aortic stent-graft. Chest. 1999;116:255-7.

19. Hollander JE, Quick G. Aortoesophageal fistula: A comprehensive review of the literature. Am J M ed. 1991;91: 297-87. Am J M ed. 1991;91:297-87.

\section{Correspondência:}

Felipe $\mathrm{N}$ asser

Rua Santa M arcelina, 177

CEP 08270-070 - São Paulo, SP

Tel.: (11) 8224.0505

E-mail: nasser.felipe@gmail.com 